\title{
Management of low-risk chest pain in ambulatory medical assessment area and the role of the heart scoring system in identifying risk
}

\author{
Authors: Khalil Hussein, ${ }^{\mathrm{A}}$ Amged Khairy ${ }^{\mathrm{A}}$ and Sarbjit Clare ${ }^{\mathrm{A}}$
}

\section{Introduction}

Low-risk cardiac chest pain at Birmingham City Hospital $(\mathrm{BCH})$ is managed through its ambulatory medical assessment area (AMAA). The pathway receives referrals from two streams, the emergency department (ED) and general practice. It follows a clear pathway, including patients being pain free with non-dynamic ECGs. Over the past 4 years we have seen the number of referrals increasing, with a large proportion of end diagnoses not being cardiac. Currently, we do not use any risk stratification tools and we present this data to promote the usage of the HEART scores ${ }^{1}$ in AMAA.

\section{Materials and methods}

This is the fourth year that this data review has been completed. We retrospectively reviewed the notes of patients referred as 'chest pain' between the dates 1 July 2019 and 31 August 2019. Patients that did not attend, self-discharged, or those with missing notes were excluded. We reviewed data regarding duration of stay, age, gender, initial diagnosis, outcome/ follow-up for patients and any adverse events within 30 days of discharge.

We have also collated data to retrospectively apply HEART scores to our patient cohort.

\section{Results and discussion}

Four-hundred and twenty-two patients' notes were reviewed. Some patients were given multiple diagnoses; $38.4 \%$ of patients were diagnosed with musculoskeletal chest pain, this being the most frequent diagnosis, and only $2.8 \%$ of patients were diagnosed with acute coronary syndrome.

$92.4 \%$ of patients were discharged from AMAA with or without follow-up. 32 patients were admitted, of which 13 were admitted under cardiology, while a further seven patients were either discussed with or reviewed by the cardiology team.

Authors: ${ }^{A}$ Sandwell and West Birmingham NHS Trust, Birmingham,
Thirty-five return events occurred within 30 days of the initial assessment; some patients returned more than once. One person underwent an inpatient angiography, but no significant disease was found. One patient had an angiography following an outpatient cardiology review. Two patients required inpatient cardiology reviews. Two patients were referred to outpatient cardiology. It was noted that one patient died, but this was unrelated to their initial assessment. One patient's notes were unavailable.

Retrospective application of HEART scores identified that cardiology involvement was $2.3 \%$ in those scoring in the lowrisk group, $18.0 \%$ in the medium-risk group and $16.0 \%$ in the high-risk group.

\section{Conclusion}

AMAA at Birmingham City Hospital continues to assess and manage low-risk cardiac chest pain safely and robustly, with low numbers being re-admitted and requiring inpatient care. Each year the numbers seen in AMAA have increased; a large proportion of these patients are diagnosed with musculoskeletal chest pain, which was traditionally managed in the ED but which is now being now being sent across to medicine.

HEART scores could be used to ensure the right patients with low-risk cardiac chest pain are being seen in AMAA. We will be adapting our pathways to incorporate the HEART score to ensure the appropriate patients follow the correct pathways, which will empower our clinicians with confidence to discharge patients.

\section{Conflicts of interest}

None declared.

\section{Reference}

1 Six AJ, Backus BE, Kelder JC. Chest pain in the emergency room: value of the HEART score. Neth Heart J 2008;16:191-6. 